\title{
Electronic Structure of Titania Nanosheets with Vacancies Based on First-Principles Calculations*
}

\author{
Yuichi Uchida ${ }^{\dagger}$ and Masahiro Hara \\ Department of Physics, Graduate School of Science and Technology, \\ Kumamoto University, Kurokami 2-39-1, Kumamoto 860-8555, Japan \\ Asami Funatsu \\ Department of Chemistry, Graduate School of Science and Technology, \\ Kumamoto University, Kurokami 2-39-1, Kumamoto 860-8555, Japan \\ Fuyuki Shimojo \\ Department of Physics, Graduate School of Science and Technology, \\ Kumamoto University, Kurokami 2-39-1, Kumamoto 860-8555, Japan \\ (Received 21 November 2017; Accepted 15 January 2018; Published 31 January 2018)
}

\begin{abstract}
Titania nanosheets (TNSs) are expected to lead to higher photocatalytic performance than bulk $\mathrm{TiO}_{2}$ because its high reactivity arises from the two-dimensional structure. While their electronic properties are considered to be easily affected by structural vacancies, the effects of vacancies in TNSs on the photocatalytic performance are not clear. In order to study them, we have performed first-principles molecular dynamics simulations for structure models of TNSs with several vacancies. First, we have calculated the electronic density of states and bandgap energies $E_{\mathrm{g}}$. It has been found that $E_{\mathrm{g}}$ of our structure modes of TNSs with vacancies are larger than bulk anatase. This tendency is consistent with the experimentally observed relationship of $E_{\mathrm{g}}$ between TNSs and anatase. It has been clarified from non-adiabatic calculations that the existence of vacancies in TNSs causes spatial charge separation and leads to longer recombination time. [DOI: 10.1380/ejssnt.2018.1]
\end{abstract}

Keywords: Density functional calculations; Molecular dynamics; Titanium oxide; Titania nanosheets; Excited states; Vacancies

\section{INTRODUCTION}

Titanium dioxide (titania, $\mathrm{TiO}_{2}$ ) photocatalysts have been intensively researched because they exhibit valuable photocatalytic properties, as typified by the harmful substance decomposition function [1], associated with ultraviolet absorption. While two crystal structures of rutile and anatase have been studied so far, titania nanosheets (TNSs) attract much interest recently. TNSs show high reactivity because their two-dimensional structure has more reactive sites [2]. In addition, TNSs allow us to design new lightweight, flexible and high-performance functional materials because their thinness permits them to be laminated with other films. For example, an enhancement of the photochemical activity of TNSs due to layer-bylayer integration with reduced graphene oxides has been reported [3]. For these reasons, it has been considered that TNS is a strong candidate for a next-generation photocatalytic material.

The chemical formula of TNS is known to be $\mathrm{Ti}_{0.87} \mathrm{O}_{2}$, which is synthesized by the delamination from a lepidocrocite-type parent layered $\mathrm{K}_{0.8} \mathrm{Ti}_{1.73} \mathrm{Li}_{0.27} \mathrm{O}_{4}$ crystal [4]. Thus, the presence of $13 \% \mathrm{Ti}$ vacancies is expected. Actually, the Ti vacancies have been directly observed by transmission electron microscopy (TEM) [5]. However, the effects of the Ti vacancies on the photocatalytic activity have not been revealed yet. In order to elucidate them, it is essential to examine the electronic ground and excited states of TNSs with the Ti vacancies.

\footnotetext{
* This paper was presented at the 8th International Symposium on Surface Science, Tsukuba International Congress Center, Tsukuba, Japan, October 22-26, 2017.

$\dagger$ Corresponding author: 163d8003@st.kumamoto-u.ac.jp
}

With regard to atomic structures around the Ti vacancies, it has been suggested that two 1-fold coordinated $\mathrm{O}$ atoms around the $\mathrm{Ti}$ vacancies tend to be removed [5]. However, the atomic configurations around the $\mathrm{Ti}$ vacancies have not been definitely determined yet. In this study, we have performed first-principles molecular dynamics (MD) simulations for several structure models of TNSs with a Ti vacancy, and investigated the effects of the Ti vacancy on the electronic structure of TNSs.

\section{COMPUTATIONAL METHODS}

\section{A. Numerical details}

Adiabatic electronic states were calculated in the framework of the density functional theory (DFT) [6] using the projector-augmented-wave (PAW) method [7], which is an all-electron electronic-structure-calculation method within the frozen-core approximation. The generalized gradient approximation (GGA) was used for the exchange-correlation energy [8]. The plane-wave cutoff energies are 30 and $250 \mathrm{Ry}$ for the electronic pseudowave functions and the pseudo charge density, respectively. The DFT plus Hubbard $U$ model $(\mathrm{DFT}+U)$ was employed in our calculations with $U_{\text {eff }}=4.2 \mathrm{eV}$ [9]. We used the $\Gamma$ point for Brillouin zone sampling.

In nonadiabatic MD simulations, electronic transitions were treated by fewest-switches surface-hopping (FSSH) method along with the Kohn-Sham (KS) representation of time dependent DFT (TDDFT). We calculated the time evolution of the density matrix with fixed atomic configuration between MD steps [10]. The elements of the density matrix determine the switching probability between 

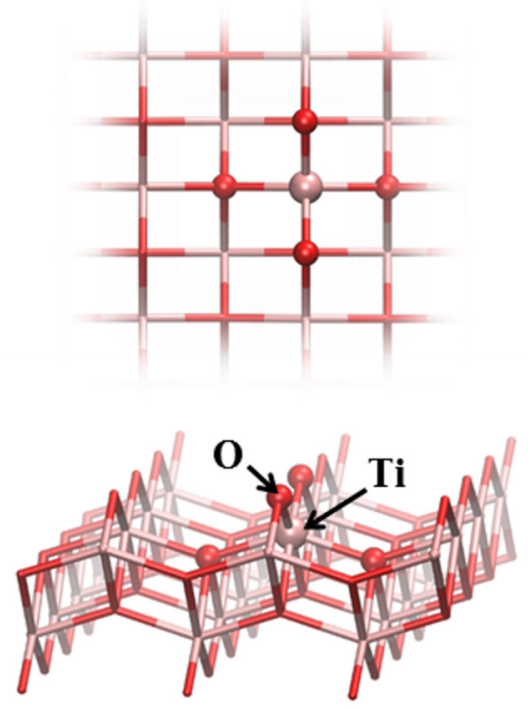

(a) Perfect model
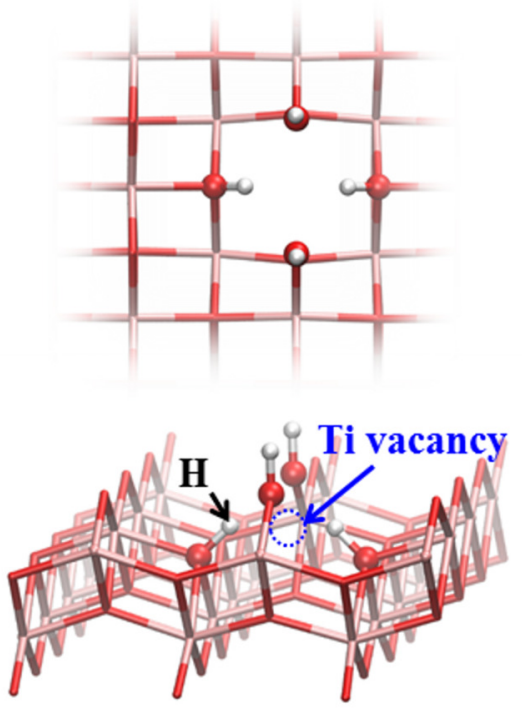

(b) TiV-H model
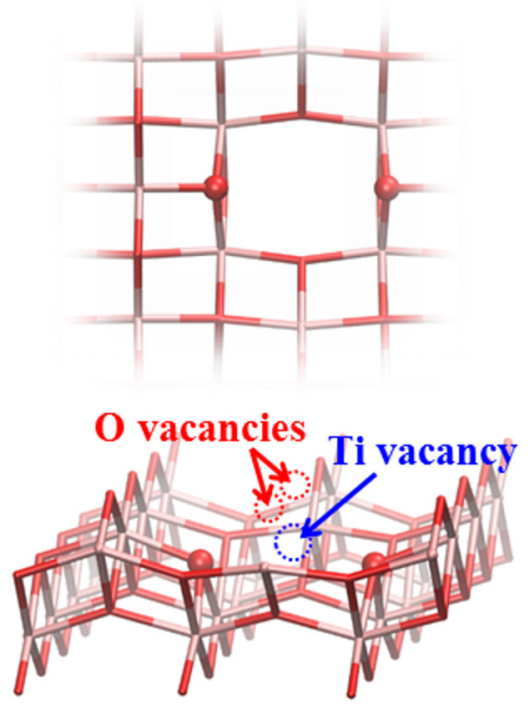

(c) $\mathrm{TiO}_{2} \mathrm{~V}$ model

FIG. 1. Optimized structures in three models. The pink, red, and white spheres represent Ti, O, and H atoms, respectively. (a) Perfect model has no vacancy. In (b) TiV-H model, one Ti atom is removed and four $\mathrm{O}$ atoms around the Ti vacancy are terminated by $\mathrm{H}$ atoms. In (c) $\mathrm{TiO}_{2} \mathrm{~V}$ model, one $\mathrm{Ti}$ and two 2 -fold coordinated $\mathrm{O}\left(\mathrm{O}_{2 \text {-fold }}\right)$ atoms bonding to the Ti atom are removed.

the adiabatic states that are expressed by a single Slater determinant. To estimate the many-body correction, we used a linear-response TDDFT of Casida [11].

We have performed simulations for the monolayer TNSs in a supercell of a dimension of $11.4 \times 18.5 \times 10.0 \AA^{3}$, under periodic boundary conditions. The TNS with no vacancy consists of $36 \mathrm{Ti}$ and $72 \mathrm{O}$ atoms. We introduced a vacuum region in the $z$ direction to avoid an interaction between TNSs. MD simulations were carried out at $300 \mathrm{~K}$, as controlled by a Nosé-Hoover thermostat. The computation times were 1210 fs and 605 fs for adiabatic and non-adiabatic calculations, respectively.

\section{B. Structure models}

We have used three models of TNSs as displayed in Fig. 1. Figure 1(a) shows the optimized structure without vacancies (Perfect model). Figures 1(b) and 1(c) show the optimized structures with vacancies. In Fig. 1(b), one Ti atom is removed and four $\mathrm{O}$ atoms around the Ti vacancy are terminated by $\mathrm{H}$ atoms (TiV-H model). In Fig. 1(c), one $\mathrm{Ti}$ atom and two 2 -fold coordinated $\mathrm{O}\left(\mathrm{O}_{2 \text {-fold }}\right)$ atoms bonding to the $\mathrm{Ti}$ atom are removed $\left(\mathrm{TiO}_{2} \mathrm{~V}\right.$ model $)$. As described in Introduction, the chemical composition of $\mathrm{Ti}_{0.87} \mathrm{O}_{2}$ has been expected from the synthesis method. However, we focus on charge neutral systems in this study, as the TEM experiments [5] have suggested that $\mathrm{O}$ atoms around a Ti vacancy tend to be removed, which may justify the $\mathrm{TiO}_{2} \mathrm{~V}$ model. The charged systems with a deficiency of Ti atoms will be studied in our future work.

In addition, we calculated the electronic states of bulk anatase to compare them with TNSs. The local structures of TNSs and bulk anatase differ from each other in the coordination number of $\mathrm{O}$ atoms. In bulk anatase, each $\mathrm{O}$ atom is coordinated to three $\mathrm{Ti}$ atoms. On the other hand, in TNSs, there are two types of O atoms (see Fig. 1).
One exists at the top or the bottom of a TNS, and is twofold coordinated to $\mathrm{Ti}$ atoms. The other exists in the middle of the TNS, and is four-fold coordinated to Ti atoms. In both TNSs and bulk anatase, all Ti atoms are six-fold coordinated to $\mathrm{O}$ atoms.

\section{RESULTS AND DISCUSSION}

\section{A. Energetic stability}

To discuss the physical properties of the structure models, it is important to estimate the relative stability of each model. The defect formation energy $E_{\mathrm{d}}$ for the $\mathrm{TiO}_{2} \mathrm{~V}$ model can be estimated by subtracting the energy per formula unit of the Perfect model from that of the $\mathrm{TiO}_{2} \mathrm{~V}$ model. The value of $E_{\mathrm{d}}$ was obtained to be 3.6 $\mathrm{eV}$, which confirms that the Perfect model is more stable than the $\mathrm{TiO}_{2} \mathrm{~V}$ model, though $\mathrm{Ti}$ vacancies are introduced in TNSs due to the synthesis method as observed in the TEM experiments [5]. The comparison of the energetic stability between the $\mathrm{TiV}-\mathrm{H}$ and $\mathrm{TiO}_{2} \mathrm{~V}$ models is also possible by calculating the energy gain or loss of the reaction, in which the $\mathrm{TiO}_{2} \mathrm{~V}$ model reacts with two $\mathrm{H}_{2} \mathrm{O}$ molecules to form the TiV-H model. We found that this reaction has the energy gain of $3.0 \mathrm{eV}$, which indicates that the TiV-H model is more stable than the $\mathrm{TiO}_{2} \mathrm{~V}$ model. As explained in Section II.B, the $\mathrm{TiO}_{2} \mathrm{~V}$ model seems to be consistent with the observation of the TEM experiments [5]. In our calculations, we did not consider the energy barriers for the reactions, which may be important to discuss which structure actually appears, and will be studied in our future work. 


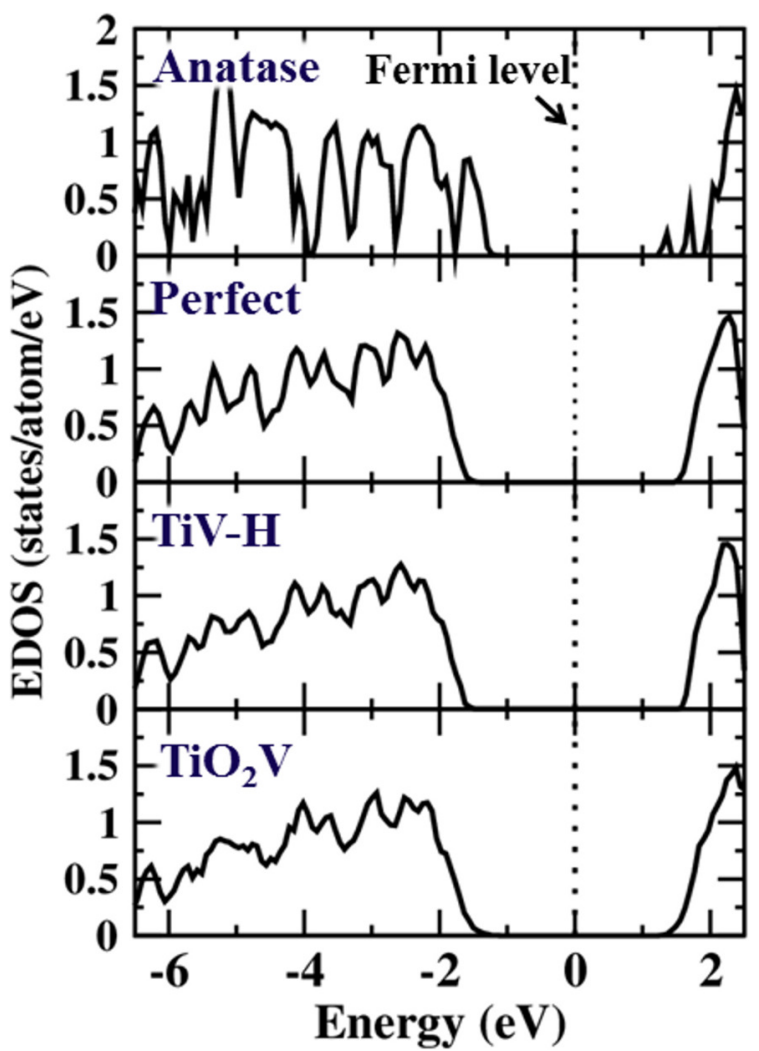

FIG. 2. Electronic density of states. The dotted line indicates the Fermi level.

TABLE I. Calculated bandgap energies $E_{\mathrm{g}}$ in the models studied.

\begin{tabular}{ccccc}
\hline \hline & Anatase & Perfect & TiV-H & $\mathrm{TiO}_{2} \mathrm{~V}$ \\
\hline$E_{\mathrm{g}}(\mathrm{eV})$ & 2.68 & 3.36 & 3.38 & 3.07 \\
\hline \hline
\end{tabular}

\section{B. Electronic density of states}

In order to study the electronic ground states, we have calculated the electronic density of states (EDOS) and bandgap energies $E_{\mathrm{g}}$ for each structure model and bulk anatase. The calculated EDOS and $E_{\mathrm{g}}$ are shown in Fig. 2 and Table I, respectively. The similarity of EDOS and $E_{\mathrm{g}}$ between the TiV-H and Perfect models indicates that the TiV-H model has a similar electronic structure to the Perfect model. This is due to few dangling bonds in the TiV$\mathrm{H}$ model. It has been reported from electrochemical and photochemical measurements that $E_{\mathrm{g}}$ of TNSs $(3.8 \mathrm{eV})$ is larger than anatase $(3.2 \mathrm{eV})[2]$. We, therefore, conclude that the atomic configurations around the $\mathrm{Ti}$ vacancies in both the $\mathrm{TiV}-\mathrm{H}$ and $\mathrm{TiO}_{2} \mathrm{~V}$ models, as well as the atomic configuration in the Perfect model, are reasonable in the sense that their $E_{\mathrm{g}}$ values are larger than anatase.

\section{Recombination time}

A recombination time of a hole and an excited electron is known as an indicator to evaluate the photocatalytic activity. We have estimated the recombination time $\tau$
TABLE II. Calculated recombination time $\tau$ in the models studied.

\begin{tabular}{lccc}
\hline \hline & Perfect & TiV-H & $\mathrm{TiO}_{2} \mathrm{~V}$ \\
\hline$\tau(\mu \mathrm{s})$ & 2.7 & 4.1 & 7.3 \\
\hline \hline
\end{tabular}

(Table II) from the Fermi's golden rule as:

$$
\tau=\left\{\frac{2 \pi}{\hbar}\left|\left\langle\psi_{\mathrm{h}}\left|H^{\prime}\right| \psi_{\mathrm{e}}\right\rangle\right|^{2} \delta\left(E_{\mathrm{h}}-E_{\mathrm{e}}\right)\right\}^{-1},
$$

where $\hbar$ is the Planck's constant, $H^{\prime}$ is a non-timedependent perturbation, and $\psi_{\mathrm{e}}$ and $\psi_{\mathrm{h}}$ are the electronic wave functions of the excited electron and the hole, respectively. $E_{\mathrm{e}}$ and $E_{\mathrm{h}}$ are the energy eigenvalues corresponding to $\psi_{\mathrm{e}}$ and $\psi_{\mathrm{h}}$, respectively.

Table II shows that $\tau$ of the $\mathrm{TiO}_{2} \mathrm{~V}$ model is the longest, and $\tau$ of the Perfect model is the shortest. It would be considered that $\tau$ of TNSs with vacancies would be shorter than the Perfect model because the vacancies might be recombination sites. However, our results are different from this consideration. In order to clarify the difference, we have investigated the spatial distribution of the hole and the excited electron.

\section{Inverse participation ratio}

To evaluate the localization of the hole and the excited electron, we have calculated inverse participation ratio (IPR) [12], which is given by the following equation:

$$
\operatorname{IPR}(\mathrm{n})=\sum_{i=1}^{N} q_{i}(\mathrm{n})^{2}, \quad(\mathrm{n}=\text { hole, electron })
$$

where $N$ is the number of atoms, and $q_{i}$ (hole) and $q_{i}$ (electron) are Mulliken charges at an atomic site $i$ obtained from the wave functions of the hole and the excited electron, respectively. IPR $=1$ and IPR $=1 / N$ represent perfectly localized and uniformly extended states, respectively. The calculated IPR values are listed in Table III.

Additionally, we have visualized the wave functions of the hole and the excited electron to clarify their spatial distribution, as shown in Table III. We see, from Table III, that the hole and the excited electron in the $\mathrm{TiO}_{2} \mathrm{~V}$ model are the most localized, and those in the Perfect model are the most delocalized. This order is the same as the recombination time. The localized hole and electron in the $\mathrm{TiO}_{2} \mathrm{~V}$ model are trapped at the atoms around the Ti vacancy and spatially separated. Consequently, it is considered that the recombination time of TNSs with vacancies becomes longer than the Perfect model.

\section{CONCLUSION}

In this study, the electronic structures of TNSs with vacancies have been investigated based on first-principles MD simulations. The obtained results are the following. (1) The TiV-H model has similar electronic structure to 
TABLE III. Calculated IPR(hole) and IPR(electron) for each model. In snapshots, the isosurfaces ( \pm 0.01 a.u.) of wave functions of the hole and the electron are shown in yellow and blue, respectively.

\begin{tabular}{|c|c|c|c|}
\hline & Perfect & TiV-H & $\mathrm{TiO}_{2} \mathrm{~V}$ \\
\hline IPR(hole) & 0.22 & 0.26 & 0.24 \\
\hline IPR(electron) & 0.09 & 0.10 & 0.27 \\
\hline $\begin{array}{l}\text { Snapshots } \\
\text { (Top view) }\end{array}$ & & & \\
\hline
\end{tabular}

the Perfect model in the ground state. (2) The TiV-H and the $\mathrm{TiO}_{2} \mathrm{~V}$ models have larger bandgap energies than bulk anatase, which is consistent with the experimental observation. (3) The existence of the Ti vacancies causes the spatial charge separation and leads to the longer recombination time.

\section{ACKNOWLEDGMENTS}

The present study is financially supported by KAKENHI [16K05478]. We would like to thank the Supercom- puter Center, Institute for Solid State Physics, University of Tokyo for the use of facilities. The computations were also carried out the computer facilities at the Research Institute for Information Technology, Kyushu University.
[1] M. R. Hoffmann, S. T. Martin, W. Choi, and D. W. Bahnemann, Chem. Rev. 95, 69 (1995).

[2] N. Sakai, Y. Ebina, K. Takada, and T. Sasaki, J. Am. Chem. Soc. 126, 5851 (2004).

[3] N. Sakai, K. Kamanaka, and T. Sasaki, J. Phys. Chem. C 120, 23944 (2016).

[4] T. Sasaki, F. Kooli, M. Iida, Y. Michiue, S. Takenouchi, Y. Yajima, F. Izumi, B. C. Chakoumakos, and M. Watanabe, Chem. Mater. 10, 4123 (1998).

[5] M. Ohwada, K. Kimoto, T. Mizoguchi, Y. Ebina, and T. Sasaki, Sci. Rep. 3, 2801 (2013).

[6] P. Hohenberg and W. Kohn, Phys. Rev. 136, B864 (1964).
[7] G. Kresse and D. Joubert, Phys. Rev. B 59, 1758 (1999).

[8] J. P. Perdew, K. Burke, and M. Ernzerhof, Phys. Rev. Lett. 77, 3865 (1996).

[9] E. Araujo-Lopez, L. A. Varilla, N. Seriani, and J. A. Montoya, Surf. Sci. 653, 187 (2016).

[10] F. Shimojo, S. Ohmura, W. Mou, R. K. Kalia, A. Nakano, and P. Vashishta, Comput. Phys. Commun. 184, 1 (2013).

[11] M. E. Casida, in: Recent Advances in Density Functional Methods, Part I, Ed. D. P. Chong (World Scientific, Singapore, 1995), p. 155.

[12] S. Blaineau and P. Jund, Phys. Rev. B 70, 184210 (2004). 